\title{
Learning the Library: Taxonomy of Skills and Errors
}

\section{Leon A. Jakobovits and Diane Nahl-Jakobovits}

\begin{abstract}
A theoretical scheme classifies user behavior into three domains of library activity-affective, cognitive, and psychomotor-and into three levels of learning-orientation, interaction, and internalization. Examples are given of library skills and errors in each of nine major classes. Applications are suggested in the areas of library orientation and instruction, testing and diagnostics, reference, signs, and guides.
\end{abstract}

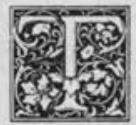

his paper presents a theory of user behavior that allows librarian to understand better what patrons feel, think and do when they use the library's resources and services. The theory's main feature is a classification scheme for cataloging the very large body of skills and errors that make up the activities of library users. Librarians will find the scheme helpful in a number of ways. Those responsible for signs and guides will learn the range of visual skills of users and the errors users may make. Staff concerned with circulation policy might consider how patrons comprehend new instructions. The preparation of orientation programs, tours, bibliographic instruction, and courses can incorporate a classification of the skills to be acquired at different levels, as well as errors to be avoided.

Consulting the taxonomy of skills and errors will facilitate the assessment of user needs and the testing of skills. Reference services can be designed to address specific user difficulties. Finally, cataloging and administrative decisions can profit from a better understanding of the actions, thoughts, and feelings of library users. In fact, all librarians could benefit by having more organized information about user behavior and a common focus through which to view their respective roles in improving patrons' use and enjoyment of the library.

\section{TAXONOMY OF USER BEHAVIOR}

Libraries are organized according to a classification scheme for books and other materials in the collection. There are many possible schemes for grouping categories of items. In our judgment it is a sign of theoretical maturity and sophistication in a profession when its practitioners come to a general agreement about a standard classification scheme.

Library science needs two kinds of classification schemes: one for books and materials, the other for user needs and behaviors. Every librarian works with both types of schemes, one of which is explicit (e.g., the Dewey Decimal Classification and the Library of Congress Classification) while the other is implicit or even unconscious. For instance, reference collections, services, and displays are accommodated to specific groups of patrons. To do this successfully, librarians must have access to certain information about the patrons, for example, their specific information needs, interests, and educational or intellectual level. This information is ordered and evaluated by the librarians making decisions, the equiva-

Leon A. Jakobovits is Professor of Psychology and Diane Nahl-Jakobovits conducts workshops in the Graduate School of Library Studies at the University of Hawaii, Honolulu, Hawaii 96822. 
lent of applying a personal classification scheme to user behavior.

Our aim is to propose a classification scheme that will allow librarians to standardize and render explicit their view of patrons' needs, skills, and errors. The taxonomy makes use of concepts fruitfully developed in educational psychology, especially the three taxonomies of educational objectives: the cognitive domain, the affective domain, and the psychomotor domain. ${ }^{1}$

\section{DEFINING THE THREE DOMAINS}

Librarians are familiar with the concept of "major classes." Schemes for classifying educational objectives, goals, outcomes, or skills and errors consist of three major classes called domains of behavior. This is the result of a remarkable consensus throughout the history of psychology and philosophy that dates to the ancient civilizations of the Hebrews and the Greeks. In this view, a human being has three parts: the soul, the mind, and the body. In the modern era this psychological trine has been described in great detail by Emanuel Swedenborg, using the concepts of the will, the understanding, and the actions. ${ }^{2}$

Swedenborg defined the will as a transcendental organ containing the affections, loves, predispositions, and strivings of an individual. The organ of understanding contains the mental processes whereby rational decisions are made, knowledges gathered, confirmations of truth or falsity established, and in which intelligence and wisdom develop. The actions of a person are carried out by the organs of the body in accordance with the thoughts and plans of the understanding and with the energy provided by the loves and purposes of the will.

Contemporary psychology has retained this fundamental threefold organization of the person. This is reflected in its history and in its tripartite professional division into dynamic psychology, cognitive psychology, and behaviorism, which study discrete zones or domains of behavior. Dynamic psychology deals with affective behavior: motivation and conflicts; feelings, character, and mood; emotional needs and inner attitudes; attractions and predispositions; the source of creativity and higher values. Cognitive psychology deals with mental problem-solving activities: plans, opinions, and beliefs; reasons and moral justifications; knowledge and comprehension. Behaviorism deals with the psychomotor domain of physical and physiological behavior: movements, sensations, perceptions, and speech. Although each of the three subdivisions has its own focus and research methodology, psychologists agree that human behavior always involves these three aspects when considered outside an experimental context.

\section{THE DOMAINS OF USER BEHAVIOR}

Learning the library involves the acquisition of habits in all three domains of behavior. In general, consider a patron or student who has a need for informatio: 1 as a result of an assignment, hobby, or personal interest. "Having an information need" is classified as an affective behavior. "Knowing where and how to find information" is a cognitive behavior. "Performing the physical steps" are behaviors in the psychomotor domain. In particular, consider a user who is looking through a periodical index and intentionally forces the eyes to run down the list of authors without skipping. "Persevering in an intention" is an affective skill. "Decoding the meaning of the content read and evaluating its relevance" are cognitive skills: the user sees a name and decides to look it up. "Hand-eye coordination, postural adjustments, and accuracy" are psychomotor skills: the user writes down the reference and resumes visual inspection of the index.

Errors, too, are committed in all three domains. At the general level, one of our students reported that he had a friend ask the librarian a question because he was too intimidated to do it himself. This is an affective error, an "unwillingness to practice library tasks." The same student spent quite a bit of time looking for books on psychology in the $P$ section, and later, by happenstance, discovered that it is the $B F$ section that contains books on psychol- 
ogy. This is a cognitive error, "making an incorrect assumption about library distinctions." This student also failed to write down the full reference of an article consulted, so that he had to return to the library; this is a psychomotor error: "not copying exactly." At this particular level, consider an individual who does not inspect a call number fully (a psychomotor error) because he figures that he only needs the first two lines (a cognitive error) and does not have the patience to learn the meaning of call numbers (an affective error).

\section{COMPLEXITY OF BEHAVIORAL TAXONOMIES}

The taxonomies of educational objectives are arranged by their creators along a continuum of progressive development, from simple to complex. This is basic to educators since the curriculum steps must match the intellectual growth of the learners. While a classification scheme can be arbitrary, a taxonomy must have an empirical validity so as to adequately represent actual growth steps. ${ }^{3}$

The categories of the LC Classification are made and unmade in accordance with the content of new books and materials. The thought processes of the user are not directly relevant in the cataloger's decision making about a particular book. By contrast, the categories of the taxonomy of library skills are representative of user behaviors and not of the characteristics of library resources.

The affective taxonomy of educational objectives has five levels of complexity: receiving or attending, responding, valuing, organizing, and being characterized. ${ }^{4}$ The cognitive domain of educational objectives has six levels: knowledge, comprehension, application, analysis, synthesis, and evaluation. ${ }^{5}$ The psychomotor domain has seven levels: perception, set, guided response, mechanism, complex overt response, adaptation, and origination. ${ }^{6}$ The Pierce-Gray classification has six parallel levels for each domain. ${ }^{7}$ According to the scheme recently reported by Hubert Dreyfus and Stuart Dreyfus, one progresses through five stages in becoming an expert at a job or hobby: nov- ice, advanced beginner, competence, proficiency, expertise. ${ }^{8}$ The Kohlberg scheme for classifying the development of moral behavior has been extensively used in empirical research. ${ }^{9}$ Many others can be cited, as this has been an active research issue since the pioneering work of Jean Piaget. $^{10}$

\section{DEFINING THREE LEVELS}

How many levels should there be and how should one decide? The creators of the affective and cognitive taxonomies of educational objectives found no satisfactory solution to this question and expect changes in the taxonomies to be made in the future. Our approach is to determine, on a theoretical basis, the minimum number of levels possible. Once this is found, our scheme provides for any number of subdivisions within each level, thus accommodating the specific requirements of other schemes.

Benjamin Bloom and collaborators support the notion of levels as a process of "internalization" or progressive deepening process. ${ }^{11}$ They cite the work of Herbert Kelman on the types of social influence that a person accepts from others. ${ }^{12}$ Three stages of increasing depth of influencing are specified: compliance (obedience from innocence or fear), identification (conformity from the desire to affiliate), and internalization (voluntary agreement from subjective freedom or personal choice). We shall draw upon these concepts to define three levels of library learning: Level 1, orientation; Level 2, interaction; and Level 3, internalization.

\section{Level 1. Orientation}

This is a stimulus-bound or concrete stage of library learning. A user is challenged to get to know the library: to memorize locations, procedures, new vocabulary. Orientation is an adjustment stage in which the person must be willing to be influenced by librarians, must have a desire to comply with instructions, and obey signs. The user goes through the motions of looking around, noticing and memorizing, pushing buttons and pulling levers, and giving cursory examination to book 
covers, inside pages, titles, names. These actions are accompanied by numerous verbalizations. Further, the prospective user must find some value and satisfaction in accomplishing the little tasks that make up the orientation or preparation phase of library learning.

At this initial level the patron has few cognitive resources to comprehend the full meaning of searching. When required to do a search for an assignment, students who are mostly active at this level (rather than at Level 2) operate from their undisciplined self-intelligence. Without an understanding of the library as a system, they lack objectivity and use inappropriate logic. They are unaware of resources, they do not consult guides, they make many guesses, they feel frustrated, they get angry, they complain, they feel alienated in the library environment, they speculate, fantasize, resist. Naturally, their effort is limited, spurious, incomplete, and fraught with error.

\section{Level 2. Interaction}

A user's negative bias toward the library is automatic. Learning the library requires the reformation of one's thinking, the reordering of one's values towards precision, systematicity, and attention to perceptual and semantic details. This is achieved by giving up uninformed selfintelligence and adopting the librarian's way of thinking in accordance with the system's way of ordering things. The individual must want to identify with the library by establishing a satisfying relationship with librarians, their services, and tools. To succeed, learners must adopt a positive bias toward the library, must trust the system, and believe in its efficacy and validity. They must derive satisfaction from their growing ability to think and act like a librarian or expert user. They must accept the new mode of thinking and actively want to extend their library competence.

When learners are active at this interaction level, they can use complex information tools and have gained an objective understanding of the library's organization. They have a continuous desire to improve their search abilities and enjoy ex- ploring on their own initiative new tools and services the library has to offer. They find it worthwhile to spend time using the library.

\section{Level 3. Internalization}

The focus at Level 1 is to know the library; at Level 2 to believe the library; at Level 3 to love the library. To operate at Level 2 there must be a reformation of the user's thinking, but to enter Level 3 the user must acquire a still more internal relationship to the library that can be characterized as moral and global. There is a feeling of congruence with library values such as conservation, service, and lifelong learning. This is an active process of incorporating the ways of the library into one's life. The user becomes a supporting patron, promoting the goals and functions of the library in society, and appreciating the library's role in the preservation of ideas and freedom. Users begin to cumulate their research experience, become knowledgeable in a field, recognize trends or patterns, and while browsing and searching they begin to have correct intuitions about likely outcomes and workable directions. There is enthusiasm and excitement at successes and discoveries and a desire to share them with others. The library system spills over into other areas of the user's daily activities: job, hobby, home management, search for health information, personal growth, research. The user becomes an information resource to family, friends, and neighbors.

\section{TAXONOMY OF LIBRARY SKILLS AND ERRORS}

We have defined and described three domains of library skills or types of user behaviors and three levels within each domain. By combining these two dimensions together into a matrix we produce a contingency table of nine zones, exemplified in table 1 . The authors of the taxonomies of educational objectives mention the notion of correlating, paralleling, or interpenetrating the three domains, though only the Pierce-Gray scheme attempts to do this in full. The idea is alive today among teachers, as shown in some of the illustrations used by Nancy Po- 
lette. ${ }^{13} \mathrm{~A}$ psychological theory of skills development capable of defining and justifying the same three levels across the three domains is required.

The contingency matrix in table 1 is an explicit theory of library skills capable of classifying all user behaviors into nine exhaustive classes. The horizontal dimension of the matrix is made up of the three discrete classes of domains of user behavior defined and reviewed above. The vertical dimension is made up of the three discrete classes of levels of development, also defined above.

In order to make use of this scheme, li- brarians must understand the three domains and the three levels and the meaning of the coordinates of the intersecting zones. The summary descriptions in table 1 are illustrative of how the zones could be described. A zone may be adequately described and paraphrased in many ways; it is important to determine accurately its features by virtue of the intersection on which it falls.

\section{SAMPLE DESCRIPTIONS FOR THE NINE ZONES}

The following are interpretations for

TABLE 1

TAXONOMY OF LIBRARY SKILLS AND ERRORS

\begin{tabular}{ll}
\hline \hline & \multicolumn{1}{c}{ Affective Domain } \\
\hline LEVEL 3 & \multicolumn{1}{c}{ A3 } \\
Affective \\
Internalization \\
Internalizing the & $\begin{array}{l}\text { Demonstrating sup- } \\
\text { port for the library } \\
\text { pibrary }\end{array}$ \\
& $\begin{array}{l}\text { perspective on soci- } \\
\text { ety and self. } \\
\text { (=library con- } \\
\text { science and moral- } \\
\text { ity versus negli- } \\
\text { gence) }\end{array}$ \\
\hline
\end{tabular}

A2

Affective

Interaction

LEVEL 2

Interacting with the library
Demonstrating continuous striving and value preferences favorable to the library and its system.

(=positive library attitudes versus library resistance)

\section{Cognitive Domain}

C3

\section{Cognitive}

Internalization

Acquiring personal knowledge and subjective intuition of a scholarly discipline. (= disciplinary connection versus lacking connection)
Psychomotor Domain

P3

\section{Psychomotor}

Internalization Performing cumulative searches in one's field and promoting the library in one's life.

(=lifelong library use versus library disuse)

\begin{tabular}{|c|c|c|c|}
\hline $\begin{array}{l}\text { LEVEL } 1 \\
\text { Orienting to the } \\
\text { library }\end{array}$ & \begin{tabular}{l}
\multicolumn{1}{c}{ A1 } \\
\multicolumn{1}{c}{ Affective } \\
Orientation \\
Demonstrating will- \\
ingness to practice \\
library tasks and \\
maintaining selec- \\
tive attention. \\
(= library adjust- \\
ment versus library \\
maladjustment)
\end{tabular} & \begin{tabular}{l}
$\quad$ C1 \\
\multicolumn{1}{c}{ Cognitive } \\
Orientation \\
Acquiring representive \\
knowledge and com- \\
prehending library- \\
relevant distinctions. \\
(=library map and \\
glossary versus library \\
ignorance)
\end{tabular} & \begin{tabular}{l}
$\quad$ P1 \\
\multicolumn{1}{c}{ Psychomotor } \\
Orientation \\
Performing physical \\
operations (hands-on \\
experience, browsing \\
and walking around). \\
(=library exploration \\
and efficiency versus \\
library avoidance and \\
inefficiency)
\end{tabular} \\
\hline
\end{tabular}

\section{$\mathrm{C} 2$}

Cognitive

Interaction

Acquiring objective knowledge of search sequences, their analysis and synthesis.

(=library search protocol versus idiosyncratic search protocol)

\section{P2}

Psychomotor Interaction Negotiating search queries and performing a single, one-time search that meets a current information need.

(=library proficiency versus library ineptitude) 
each zone with examples of skills and errors. Note that the definition of each zone is given by the intersection, which remains standard or fixed (e.g., affective orientation versus affective interaction; $c o g-$ nitive orientation versus cognitive interaction, etc.). But the description of each zone and the specific examples permit variable content, as long as the description is congruent with the fixed criteria of the definition. In this case, in addition to the description, a title is given for each zone in table 1 (e.g., "Positive Library Attitudes vs. Library Resistance" for zone A2 or, "Lifelong Library Use vs. Library Disuse" for P3). Other titles could be chosen that paraphrase the same content.

\section{A1. Affective Orientation}

This phase is entitled "Library Adjustment vs. Library Maladjustment" to reflect the motivational challenge a new user must meet when learning to interact with the library. Given the human law of least effort, one is required to compel oneself to do what at first appears to the novice as mere busy work. For instance, we observed a hapless student, alarmed, comment to a librarian who was showing him a catalog drawer: "Oh, no! You mean I have to go through all of that?" Upon which the librarian answered, "Searching is work. It takes time!" It takes a certain degree of psychological or emotional adjustment to be ready and willing to practice detailed and systematic tasks given to one by another person, often unexpectedly, and to maintain one's selective attention continuously for some minutes or hours. Some examples: User is willing to look at a library map until it becomes clear; accepts a librarian's suggestion; shows assertiveness and perseverance in completing a hands-on assignment.

These behaviors indicate a patron's affective orientation toward learning the library. People who are active in this zone demonstrate task perseverance and compliance, or their opposites, unwillingness to practice and to focus attention on the specialized stimuli of the library. Given the primacy of the affective domain it is to be expected that individuals who are ei- ther not active, or negatively active, in this zone will experience emotional conflict and resistance in attempting to acquire search skills. Some examples: User feels ashamed to be seen re-using services; has another person ask the librarian a question; has undecipherable notes after a search; has compulsive thoughts of being attacked in the library; and would rather be elsewhere.

\section{C1. Cognitive Orientation}

The main feature of this zone is memorizing library-relevant distinctions such as books versus magazines, current periodicals versus bound volumes, subject cards versus author or title cards, regular stacks versus reference, online catalog versus comcat, etc. New terminology is learned. Mental maps are established. New but fairly simple procedures become familiar. This phase is entitled "Library Map and Glossary." Some examples: User can interpret the parts of a catalog card; can distinguish between citation and abstract; comprehends shelf locations by subject; and can phrase a reference query. The learning in this zone is representative of the actual behaviors to be carried out during a search; for this reason we call it "representative knowing," to be distinguished from "objective knowing," described in $\mathrm{C} 2$ below.

Cognitive orientation in library learning is made up of mental verbalizations that aid in the manipulation of a sequential task such as systematic browsing or locating a book by its call number. It involves memory, association, and common sense. These mental verbalizations, or self-talk, serve as the basis for a higher type of mental verbalization called "search protocol" (zone C2), which no longer follows common sense but the specialized reasoning of the library system. Individuals who are inactive in cognitive orientation (-C1) remember little, see little, and comprehend little ("Library Ignorance"). They fail to lay the foundation for search skills.

\section{P1. Psychomotor Orientation}

This zone of learning is titled "Library Exploration and Efficiency vs. Library Avoidance and Inefficiency." Its main fea- 
tures are physical movements, visual scanning, and external verbalizations. Prospective users require a phase of adjustment to the library environment, and a significant component of this adjustment is acquired through direct exploration of locations, shelves, signs, and the manipulation of drawers, levers, and buttons on machines. Some examples: User tries microfiche reader to see how it works; browses through the two Library of Congress Subject Headings volumes; does assigned tasks on online catalog; takes a walking tour of the library; copies a call number correctly; asks a question; verbalizes thoughts out loud; blushes; hesitates; repeats a definition. Psychomotor orientation errors, "Library Avoidance and Inefficiency" (-P1), include: User does not allocate time for learning to use the library; scans screen on microfiche reader instead of first looking at the index on the fiche to locate the matrix coordinates; commits alphabetical errors in searching, ending up in the wrong drawer or on the wrong fiche; records a call number incompletely; does not allocate sufficient time for library searching; and looks up author under first name instead of last name.

While these psychomotor behaviors are executed, cognitive and affective orientation occur simultaneously, on both a general and a specific level. For instance, in the activity "User manipulates microfiche reader," there is a general motivation to learn a new tool and numerous specific motivations or intentions in the subtasks of selecting a fiche, inserting it in a particular way, and viewing different parts of it.

We note here the importance of verbalizations as a psychomotor behavior that involves speech mechanisms. Other taxonomies on the psychomotor domain have not included overt speech responses in their classification scheme, but we believe that to do so is consistent with the nature of the three domains. At the lowest level, verbalizations in the form of instructions, signs, definitions, or warnings are stored in memory and utilized by merely repeating them at the right time as a means to help guide one's behavior. As noted by B. F. Skinner and ly L. S. Vygotsky, overt verbalizations are character- istic of children performing tasks. ${ }^{14}$

As age advances, verbalizations become less intense; in the adult stage they no longer occur except in instructional situations where an adult is learning something totally new. Verbalizations are to be distinguished from the cognitions and affections that precede or cause them. For example, reading a sign involves a psychomotor behavior (P1), but comprehending its meaning is a cognitive behavior (C1). External verbalizations at a higher level are expressions of inner affections, as when a "User expresses gratitude upon accomplishing a successful search" (P3).

External verbalizations, which are psychomotor, are to be distinguished from internal verbalizations, which are cognitive. The two correspond, as observed by Anders Ericsson and Herbert Simon. ${ }^{15}$

\section{A2. Affective Interaction}

This zone of library learning, "Positive Library Attitudes," concerns the user's values regarding the library. While affective orientation (A1) is the willingness, through compliance or obedience, to carry out the librarian's direct instructions, affective interaction is the user's willingness to follow self-instructions out of a desire to acquire library proficiency. These two affective states are not necessarily related. For instance, a student may be willing to carry out the tasks assigned in an introductory library course (A1), but may have a negative attitude toward libraries and be unwilling to conduct a search for some personal information need. Students who drop a course because it requires library assignments also commit this affective error $(-\mathrm{A} 2)$.

When learners are active in zone A2, they have the desire to adopt the thought process of librarians. To develop library proficiency, users must cultivate a new motive, namely, the determination to face the challenges of a serious, full-fledged search. With this new-found purpose they are likely to overcome the inner forces of doubt, disbelief, or scorn; with this victory comes the beginnings of trust in the library-an essential ingredient for acquiring search competence. At last, the user seeks to identify and to interact with the 
library and its system and appreciates the opportunity to do so. This is the essential prerequisite for acquiring objective (formal, standardized) knowledge of the library. Some examples: User strives to learn new tools; resolves to be careful; wants to read library books; feels it is advantageous to learn to use the library; feels happy in a growing ability to carry out searches.

Individuals who are negatively active in this zone experience "Library Resistance" $(-\mathrm{A} 2)$, which is the absence of trust and the refusal to alter one's way of thinking as required. Some examples: User is convinced in advance that the library has nothing on a certain topic; doubts the correctness of a search sequence when it is accurate; and feels discouraged throughout a search.

\section{C2. Cognitive Interaction}

This is called "Library Search Protocol" because the objective for the learner is to be able to produce a protocol, or formalized search procedure, that makes use of information tools: subject headings, catalogs, bibliographies, and indexes-and their appropriate interlinking in a sequential search. While the earlier phase of cognitive orientation ( $\mathrm{C} 1$ ) is a representative knowing, cognitive interaction is an objective knowing that comes only from the experience of carrying out a hands-on search in response to an actual information need.

In cognitive orientation (C1), libraryrelevant distinctions are acquired by rote memory. They remain empty of objective content until the identification level is reached through $\mathrm{A} 2$ and an actual search sequence is undertaken in P2. Some examples: User continually analyzes own search procedure; prompts self with questions while searching; realizes signs must be read and heeded; reflects on ways of improving future searches; decides not to follow up on a particular citation; selects a keyword from a title to search with; reviews mental verbalizations of a search sequence in order to decide on the next step; and clusters book citations by their call number and employs this as a criterion for selecting or rejecting titles.

Cognitive interaction errors (-C2) involve the use of nonstandard or "Idiosyn- cratic Search Protocol." Some examples: user thinks of looking in the card catalog for a periodical article title; is unable to read coded citations in periodical indexes; does not understand the difference between publication history and holdings information in catalog entries; has incorrect conception of library tasks.

The level of search activity between $\mathrm{C} 1$ and $\mathrm{C} 2$ has been recognized by librarians such as James Rice, who advocates the "teaching of principles as well as techniques." ${ }^{\prime b}$ Understanding principles includes comprehending bibliographic organization, literature structure within disciplines, interactive retrieval tools such as subject headings and cross-references, and the components of a search strategy. We shall see below that one of these principles, the structure of literature within disciplines, involves $\mathrm{C} 3$ activities.

Raising the activity level from mere representative knowing (C1) to objective knowing (C2) involves the difficult challenge of learning to think along the lines of the library's organization. The success of this cognitive interaction with the system is conditioned by the individual's inner affective interaction with the library (A2): is it the case that the "user demonstrates value preferences favorable to the library" or that the "user is averse to producing mental verbalizations that involve subject headings'"? (-A2) Mental aversion to thinking in terms of packaged and crossreferenced information must be sufficiently overcome before the individual can produce an appropriate search protocol. We have suggested elsewhere how librarians might influence the affective learning of patrons. ${ }^{17}$

\section{P2. Psychomotor Interaction}

The steps of library research are classified here; hence, an appropriate title is "Library Proficiency." The physical operations carried out during psychomotor orientation (P1) activities are only "exploratory" and are not conducted under the actual motive of a search. In addition, the external verbalizations during psychomotor orientation (P1) are simply representative sentences, fixed repetitions, and imitations. In contrast, psychomotor in- 
teraction (P2) involves a higher, more objective type of talk. The user is now in an authentic searching state (Level 2), rather than in simulated exploration (Level 1) and is responding to an actual information need that may have life consequences.

The external verbalizations of Level 2 search activities are objective and resemble those of the librarian, as at the end of the negotiation process between the reference librarian and the patron. ${ }^{18}$ In P2 ("'Library Proficiency vs. Library Ineptitude"'), the steps carried out begin to count as real search experience, hence as "proficiency." Note that the process of library interaction (Level 2) is necessary in all three domains. Interacting at the psychomotor level (P2) is to verbalize like librarians about titles, subjects, and indexes, to move around like librarians or expert searchers, to walk and stand as they do in relation to shelves and areas, and to use the eyes as they do-selectively noticing details, checking items rapidly and thoroughly. Some examples: User reconciles information by continuous matching and comparing; selects correct subject headings; puts call numbers in order before going to the stacks; formulates a reference query; writes down citations; writes down synonyms for a subject; expresses appreciation to librarian for help received; and consults the reference librarian about an ongoing search.

Psychomotor interaction errors (-P2) plague and frustrate many students, as we have learned from their self-reports. Some examples: User overlooks detail previously pointed out; ignores vocabulary distinctions taught; underestimates the time a search requires; gives up a search prematurely and leaves the library without any references; and comes to the library without clothes warm enough for an airconditioned library.

\section{A3. Affective Internalization}

Many patrons, students, and faculty who are active at Level 2 remain inactive at Level 3. They are barred from further internalizing their intellectual skill by an insufficient affective commitment to the idea of the library (A3). The inability to feel love for the library is an affective error at the third level (-A3). To overcome this block, users must find in themselves generalized affections for the good, the true, and the beautiful. All educated people have stirrings of these feelings in their civilized consciences. At Level 3 the user connects these generalized subjective and idealistic feelings specifically to the library. The library perspective on society and self is loved (Level 3 ) and not just believed (Level 2) or merely known (Level 1).

This zone is titled "Library Conscience and Morality" since' it involves higher human values. For instance, we gave a onetime slide show presentation on book conservation to a social psychology class. Many students responded with spontaneous expressions of sympathy with the striving to save books from destruction by age, humidity, vermin, and careless users dropping books, forcing them open against copy machines, eating while reading, and pulling books off the shelf by the spine cap. The presentation elicited overt expressions of recognition and consequent confessions of guilt. Many promised to reform, but some insisted that the library should replace books more often! Other examples: User feels the imperative to replace a book on the shelf; feels attracted to books and libraries; accepts the idea that a library book is publicly owned; feels awe at all that books symbolize in the history of civilization; feels confident that library resources can improve the quality of life; and has feeling of peace and solitude while searching.

Negative affections at Level 3, or "Library Negligence" (-A3), include hostility toward librarians, books, and users themselves as searchers. Some examples: User dissociates self from libraries; condones self-chastisement for search errors; feels library books are not of great value since they are replaceable; feels library books are public property so they don't have to be treated carefully; doesn't care about other patrons and doesn't mind making noise.

\section{C3. Cognitive Internalization}

The patron who has acquired library proficiency knows library protocol objectively and from experience (C2); but this 
will not necessarily affect thinking in a general way. There must be a cumulative context in order for objective knowing to metamorphose into personal knowing, such as the knowing of a graduate student or faculty member who is attached to some scholarly discipline by profession or serious hobby. We have named this zone of library learning "Disciplinary Connection," since only thus can library skills be fully internalized. A discipline or field of knowledge provides keywords, subject headings, and thesauri. Through study a person perceives the importance of accurate citations and balanced bibliographies and appreciates both the complexity and the value of citation indexes.

A disciplinary affiliation or "major" affords even the undergraduate the intellectual opportunity to acquire subjective intuitions of a scholarly discipline: perceiving trends, recognizing norms, sensing standards, feeling loyalty toward a theory but defending another's right to have a fair hearing. Some specific examples: User understands how search tools facilitate finding information; can rank reference works; senses that some of the titles retrieved might be "false drops"; perceives the relevance of an annotation in a bibliography; can see how a new tool can aid in keeping abreast of new developments; and has accurate expectations about the content of a citation by considering its place of publication.

When a user is "Lacking in Disciplinary Connection" (-C3) there is little activity in Cognitive Internalization. User does not understand citation networks; holds incorrect assumptions about particular subject heading content; has no intuitions about the significance of classic works; and fails to see the importance of accurate referencing.

\section{P3. Psychomotor Internalization}

This is the ultimate zone of learning the library and integrating it into one's life. Hence it is called "Lifelong Library Use vs. Library Disuse." Users in this phase are daily information consumers, and have a continuous appetite for information. They are aware of how little they know compared to "what's out there."
Users in this zone are overt supporters of libraries, express enthusiasm for its organizational features, and experience a deep sense of satisfaction at their expertise and success in keeping abreast of new information. Users also verbalize their enjoyment and appreciation of libraries. Some examples: User expresses delight upon accomplishing a search; refrains from marking up a book; reports damage so that it may be repaired; serendipitously discovers a reference needed for another purpose; promotes the library; observes library bans on food and drink; expresses a desire to read books encountered while searching.

"Library Disuse" involves making psychomotor internalization errors (-P3), for example: User is upset in the process of using the library; swears or expresses selfcondemnation while searching; presses books flat on the photocopying machine; marks up books.

\section{SIMILARITY TO OTHER MODELS}

The theory of library behavior here presented may be helpful to librarians in promoting library use. The proposed classification scheme for user behavior allows librarians to take an integrated view of levels of library learning.

The notion that there are "levels" of development in learning the library is intuitively evident and conforms to the accepted view of learning in other fields, such as biology, psychology, and education. Nigel Ford believes that "the library may also have a distinctive role to play in producing certain types of learning" and refers to three overlapping work styles of a library learner that correspond to the three levels of the taxonomy: "dependence" (Level 1); "independence" (Level 2); and "interdependence" (Level 3). ${ }^{19}$

Enhancing library skills from Level 1 to Level 2 is, to Ford, crucial. He suggests teaching approaches that provide guidance "appropriate to particular types and levels of problems requiring information. ${ }^{20}$ To help internalize library skills, the library environment ought to encourage "affective and longer-term goals such as continuing motivation," "continuing personal development," "'allowing access 
to a variety of different approaches to, and points of view on, particular topics. ${ }^{\prime 21}$ In the taxonomy, these user behaviors are classified at Level 2. Library teaching at Level 1, to dependent learners, is by necessity more direct and immediate. Beyond orientation, library teaching becomes more indirect, involving the selection of tools, the choice of access language, and the availability of services. At Level 2 (interacting with the library), the user is relatively independent and is able to operate from affections and cognitions modeled on the role of the librarian. Level 3 development is less frequently discussed in the literature on library instruction but will receive more attention in the future.

Earlier taxonomies of educational objectives have focused on the vertical continuum of levels within each domain one at a time, thus arriving at various numbers of levels arranged on a continuum. In contrast, this proposal focuses on the horizontal integration of each level, simultaneously across the domains. This model is theoretically distinct from the concept popularized by Bloom, Krathwohl, and colleagues and differs in several major ways.

The Pierce-Gray classification system is the most elaborate and explicit of the taxonomy schemes in the Bloom tradition. ${ }^{22}$ It organizes objectives in the three domains along six semiparallel levels. Lower skills are assumed to be distinct across the three domains. For example, focusing (for cognitive), emotive imprinting (for affective), and sensory transmission (for psychomotor). Higher skills, however, are assumed to be progressively more cognitive in all three domains. According to the authors, "the degree of cognitive effort has been used as the basic sorting factor to define levels. "23 In conformity with this assumption they place the cognitive domain in the first column of their matrix, followed by affective and psychomotor ("CAP") ${ }^{24} \mathrm{Fi}$ nally, there is no integrated title for each level across the domains.

In contrast, the model proposed here orders the domains from affective to cognitive to psychomotor, in accordance with the traditional threefold view of the per- son composed of the will, the understanding, and the actions or uses. ${ }^{25}$ The will (affective domain) is first, since actions originate in it. The understanding (cognitive domain) mediates. The actions or uses (psychomotor domain) are external results or effects of the first two. The three domains are distinct, but correspond at all levels. The three domains are also explicitly integrated by level, as indicated by the single title for each level. Table 1 may be viewed as a map of the library learner's states rather than sequential stages. When viewed thus, the matrix represents a simultaneous display of a user's degree of activity within each of the nine zones.

We believe that future research will demonstrate the advantages of this type of model, which offers a simultaneous display of the lifelong involvement of an individual's library-related activities. This display may be useful to librarians in all specialties since it maps possible user behavior. The matrix can thus be used to chart the progress of library learners by determining the degree of activity of a person at any one time across the nine zones.

\section{SOME ADVANTAGES OF THE TAXONOMY AND ITS APPLICATIONS}

The main advantage of a theory in a scientific field is that it can afford a better understanding of the field's phenomena. We list below a few applications of the classification scheme presented and the theory on which it rests.

The theory

1. offers a unified focus and language for all of library science;

2. guides research, makes it cumulative, and provides testable hypotheses on user behavior, library instruction, and library policy;

3 . links library science to the social and behavioral sciences, including educational psychology and linguistics;

4. offers a check on the breadth and depth of a curriculum, course, or presentation; allows library instruction efforts to incorporate higher levels of user behavior;

5 . helps to standardize tests of library skills, to ensure balanced coverage; suggests ways tests can be used to assess the 
type of instructional efforts needed;

6. helps librarians in bibliographic instruction by allowing them to develop objectives at the appropriate level and by providing examples of the relevant language to be used for each domain;

7. provides the basis for a universal catalog of user behavior, or a national classified inventory of library skills and errors, and, possibly, a data bank that would collect and publish the cumulative observations of librarians and information specialists; in addition, it could generate an international catalog of user behavior that transcends language-specific differences in favor of general, species-related psychological standards of searching.

\section{REFERENCES}

1. Benjamin S. Bloom, ed., Taxonomy of Educational Objectives: The Classification of Educational Goals. Handbook I: Cognitive Domain (New York: David McKay, 1956); David R. Krathwohl, Benjamin S. Bloom, and Bertram B. Masia, Taxonomy of Educational Objectives: The Classification of Educational Goals. Handbook II: Affective Domain (New York: David McKay, 1964); M. David Merrill, "Psychomotor Taxonomies, Classifications, and Instructional Theory," in The Psychomotor Domain: Movement Behavior, ed. Robert N. Singer (Philadelphia: Lea and Febiger, 1972); Walter D. Pierce and Charles E. Gray, Deciphering the Learning Domains: A Second Generation Classification Model for Educational Objectives (Washington, D.C.: Univ. Pr. of America, 1979); Anita J. Harrow, A Taxonomy of the Psychomotor Domain (New York: David McKay, 1972).

2. Emanuel Swedenborg, Rational Psychology (Philadelphia: Swedenborg Scientific Assn., 1742; 1950).

3. Bloom, Cognitive Domain, p.17.

4. Krathwohl, Affective Domain, p.176.

5. Bloom, Cognitive Domain, p.201.

6. Merrill in Singer, Psychomotor Domain, p. 389-91. Merrill reports a number of other schemes in the psychomotor domain. See p.385ff.

7. Pierce and Gray, Learning Domains, p.201.

8. Hubert L. Dreyfus and Stuart E. Dreyfus, Mind over Machine: The Power of Human Intuition and Expertise in the Era of the Computer (New York: The Free Pr., 1986), p.16 ff.

9. John C. Gibbs and Keith Widaman, Social Intelligence: Measuring the Development of Sociomoral Reflection (Englewood Cliffs, New Jersey, Prentice-Hall, 1982), p.16 ff.

10. Jean Piaget, The Moral Judgement of the Child (New York: Free Pr., 1932).

11. Bloom, Cognitive Domain, p.29.

12. Krathwohl, Affective Domain, p.31-32.

13. Nancy Polette, The Research Book for Gifted Programs K-8 (O'Fallon, Mo.: Book Lures, 1984), p.151.

14. B. F. Skinner, Verbal Behavior (New York: Appleton-Century-Crofts, 1957); Lev Semenovich Vygotsky, "Thought and Word," in Thought and Language (Cambridge, Mass.: M.I.T. Pr., 1962), p.119-53.

15. K. Anders Ericsson and Herbert A. Simon, Protocol Analysis: Verbal Reports as Data (Cambridge, Mass.: M.I.T. Pr., 1984), p.239.

16. James Rice, Jr., Teaching Library Use: A Guide for Library Instruction (Westport, Conn.: Greenwood Pr., 1981), p.42.

17. Diane Nahl-Jakobovits and Leon A. Jakobovits, "Managing the Affective Micro-Information Environment," Research Strategies 3:17-28 (Winter 1985).

18. Gerald Jahoda and Judith Schiek Braunagel, The Librarian and Reference Queries: A Systematic Approach (New York: Academic Pr., 1980), p.137-39.

19. Nigel Ford, "Towards a Model of 'Library Learning' in Educational Systems," Journal of Librarianship 2:247-60 (Oct. 1979).

20. Ibid., p.256.

21. Ibid., p. 256.

22. Pierce and Gray, Learning Domains.

23. Ibid., p.183.

24. Ibid., p.201.

25. Swedenborg, Rational Psychology, p.378-97. 